donde se cultiva y asilvestra con facilidad (Morales, 1986), de floración primaveral (Abril-Junio), y la subsp. aestivus (Reuter ex Willk.) A. Bolós \& O. Bolós, endemismo de la región levantina (Castellón, Valencia, Alicante, Ibiza), citado también en Murcia (Esteve 1972, sec. R. Morales 1986) y de floración preferentemente estival (Mayo-Octubre).

Recientemente se ha localizado una población de Thymus vulgaris subsp. vulgaris L. en Cádiz, Chiclana de la Frontera, cerca de la playa, frente a una zona de dunas y bajo un pinar de Pinus pinea L. (22-III-1997, Martín Mosquero SEV 149255) lo cual supone una novedad para la Flora de Andalucía Occidental.

Hay que destacar que el terreno en el que se localiza dicha población parece haber sido removido, lo que ha podido favorecer la presencia de este taxón; aunque en la actualidad parece bien asentada.
AGRADECIMIENTOS. El autor agradece al Dr. Ramón Morales Valverde la revisión del material, así como sus orientaciones y sugerencias.

\section{BIBLIOGRAFÍA}

JALAS, J. -1972- Thymus L. In: T. Tutin et al. (eds.) Flora Europaea 3: 172-182. Cambridge.

MORALES VALVERDE, R. -1986- Taxonomía de los géneros Thymus (excluida la Sec. Serpyllum) y Thymbra en la Península Ibérica. Ruizia 3.

MORALES VALVERDE, R. 1987- Thymus L. In: B. Valdés et al. (eds.), Flora de Andalucia occidental 2: 441-446. Barcelona.

Aceptado para su publicación en Octubre de 1997

Dirección del autor. Departamento de Biología Vegetal y Ecología. Apdo. 1095. 41080, Sevilla.

\title{
62. APORTACIONES A LA FLORA DE CÓRDOBA
}

\author{
Rafael PINILLA, Rafael TAMAJÓN y Jesús M. MUÑOZ
}

New records for the flora of Cordoba

Palabras clave. Corología, Córdoba, Andalucía, España.

Key words. Chorology, Cordoba, Andalusia, Spain.

Se aportan nuevas localidades de la provincia de Córdoba, de interés corológico, para un total de 53 especies. En su mayoría proceden del Parque Natural "Sierra de Hornachuelos". Este espacio natural protegido tiene una extensión de unas 65.000 hectáreas,

Estudio financiado por la Consejería de Medio Ambiente de la Junta de Andalucía, Proyecto de Investigación: «Cartografía y Evaluación de la vegetación del Parque Natural Sierra de Hornachuelos». 
y se localiza en el noroeste de la Provincia de Córdoba, en plena Cordillera Mariánica, formando parte de la comarca de Sierra Norte. No obstante, en su parte más meridional contacta con la comarca de la Vega.

Para cada taxon se menciona la distribución previamente conocida en Andalucía Occidental, de acuerdo con la Flora Vascular de Andalucía Occidental (Valdés et al., 1987), y diversas publicaciones con posterioridad a dicha obra.

El material herborizado se encuentra depositado en el herbario del Departamento de Biología Vegetal y Ecología, División de Botánica, de la Facultad de Ciencias de Córdoba (COFC).

\section{Cheilanthes tinaei Tod.}

CÓRDOBA: Santa Eufemia, Sierra de Santa Eufemia, UH-37, 29-12-94, R. Tamajón \& R. Pinilla, COFC 24394.

Nueva cita para la comarca de Los Pedroches de este taxon rupícola. Estaba citado de las comarcas: Aracena, Andévalo y Sierra Norte (Cabezudo \& Salvo, 1987). Se ha localizado en roquedos, sobre cuarcitas silúricas. Rara.

\section{Illecebrum verticillatum $\mathrm{L}$.}

CÓRDOBA: Villaviciosa, $\mathrm{A}^{\circ}$ Pajaroncillo, UH-10, 10-6-93, R. Tamajón \& R. Pinilla, COFC 22814.

Se trata de la primera cita para Sierra Norte. Con anterioridad se conocía de Aracena, Andévalo, Campiña de Huelva, Condado-Aljarafe, Litoral, Marisma, Campiña Baja gaditana, Algeciras (Devesa, 1987), Pedroches y Zújar (Jiménez \& Ruíz de Clavijo, 1990). Se ha encontrado en suelos encharcados, sobre sustratos silíceos. Rara.

\section{Loeflingia baetica Lag.}

CÓRDOBA: Santa Eufemia, $A^{0}$ del Sotillo, UH-37, 20-4-94, R. Tamajón \& R. Pinilla, COFC 24420 .

La población localizada en la Comarca de Los Pedroches constituye una nueva cita para la misma, conociéndose hasta el momento sólo de Sierra Norte, Condado-Aljarafe, Litoral y Campiña Baja gaditana (Devesa, 1987). Esta especie se ha encontrado en pastizales sobre suelos arenosos silíceos. Poco frecuente.
Moehringia pentandra J. Gay

CÓRDOBA: Hornachuelos, Finca «Vista Alegre», TG-98, 22-5-92, R. Tamajón \& R. Pinilla, COFC 21655; Finca «El Jardín de La Aljabara», UH-00, 1-7-92, R. Tamajón \& R. Pinilla, COFC 21741; Almodóvar, "Cerro de la Viña», Finca "Berracosillas", UH-10, 25-5-94, R. Tamajón \& R. Pinilla, COFC 24411.

Nueva cita para Sierra Norte. Con anterioridad se conocía de Aracena, Litoral, Grazalema y Algeciras (Talavera, 1987). Especie indiferente edáfica, se ha herborizado en el estrato herbáceo de madroñales y de alisedas. Poco frecuente.

Tuberaria macrosepala (Cosson) Willk.

CÓRDOBA: Villaviciosa, Finca «Fuente Vieja», UH-10, 29-6-92, R. Tamajón \& R. Pinilla, COFC 21683.

Primera cita para Sierra Norte cordobesa. Con anterioridad se conocía de Aracena, Sierra Norte sevillana, Litoral y Algeciras (Gallego, 1987), y más recientemente, de la comarca del Zújar (Jiménez \& Ruíz de Clavijo, 1990). Rara.

\section{Lepidium latifolium $\mathrm{L}$.}

CÓRDOBA: Córdoba, Río Guadalquivir, «Sotos de la Albolafia», UG-49, 29-4-95, R. Tamajón, R. Pinilla \& F. Obregón, COFC 24401.

Se trata de la primera cita para la Vega. Esta especie de distribución restringida se conocía con anterioridad de la Campiña Baja gaditana, Campiña Alta y Grazalema (Clemente \& Hidalgo, 1987). Se ha herborizado en herbazales sobre sustratos aluviales, en suelos húmedos de vega. Rara.

\section{Erica umbellata $\mathrm{L}$.}

CÓRDOBA: Villaviciosa de Córdoba, Finca «Fuente Vieja», UH-10, 13-12-92, R. Tamajón \& J.M. Muñoz, COFC 21431; Hornachuelos, Finca «Mosqueros», TH-90; 26-12-91, R. Tamajón \& R. Pinilla, COFC 21450; Finca «El Asiento» TH-90, 16-1-92, R. Tamajón \& R. Pinilla, COFC 21448; Finca «Mata Román», TH-90, 27-2-92, R. Tamajón \& M. Carrasco, COFC 21433; Finca «Chamiceros», TH-90, 27-2-92, R. Pinilla \& J.M. Quero, COFC 21432; Almodóvar, Finca «El Jardín de la Aljabara», UH-00, 21-1-92, R. Tamajón \& S. Bascón, COFC 21451.

Aunque no se trata de una nueva cita para Sierra Norte cordobesa, pues ya se conocía de esta 
comarca (Fernández \& Domínguez, 1991), con estas nuevas localidades se amplía notablemente el área de distribución conocida. E. umbellata L. se presenta en nuestro territorio exclusivamente como compañera constante de jarales-brezales. También se ha detectado en jarales pringosos con Ulex eriocladus y cantuesales. Localmente abundante.

\section{Rosa sempervirens $\mathrm{L}$.}

CÓRDOBA: Hornachuelos, Río Guadalora, UH-40, 13-5-93, R. Tamajón \& R. Pinilla, COFC 22773.

Nueva cita para la comarca Sierra Norte. Hasta el momento se conocía de Algeciras, Vega, Alcores, Campiña (Alta y Baja) y Grazalema (Devesa, 1987). Se trata de un rosal localmente abundante, que alcanza su desarrollo óptimo en bosques y matorrales riparios más o menos termófilos. No obstante, la población que aquí se cita se ha encontrado en un cañaveral de Arundo donax L.

\section{Rosa canina L.}

CÓRDOBA: Belalcázar, $\mathrm{A}^{\circ}$ de la Dehesa, UH06, 22-6-94, R. Pinilla \& R. Gallardo, COFC 24384.

Nueva cita para la comarca de Los Pedroches. Con anterioridad esta especie estaba citada de Aracena, Andévalo, Sierra Norte, Litoral, Campiña Alta y Subbética (Devesa, 1987). Se ha herborizado en matorrales riparios. Poco frecuente.

\section{Sanguisorba hybrida (L.) Nordborg}

CÓRDOBA: Hornachuelos, Finca «Cuchillares», TG-99, 22-4-92, R. Pinilla \& J.M. Quero, COFC 21712; Finca «Nava Los Corchos Altos», TG-99, 9-3-93, R. Tamajón \& R. Pinilla, COFC 21713; Almodóvar, Finca «Los Lagares», UG-29, 6-5-92, R. Tamajón \& R. Pinilla, COFC 21711.

Esta es la primera cita de este endemismo mediterráneo-iberoatlántico para Sierra Norte cordobesa. Estaba citada de Aracena, Andévalo, Litoral onubense, Campiña Alta sevillana, Subbética sevillana y Grazalema (Devesa, 1987), así como de Los Pedroches (Jiménez \& Ruíz de Clavijo, 1990). Se ha herborizado en el sotobosque de alcornocales y encinares, tanto sobre sustratos silíceos como calcáreos. Poco frecuente.

Aphanes maroccana Hylander \& Rothm. CÓRDOBA: Carretera Torrecampo-
Conquista, Río Guadalmez, UH-65, 18-3-94, R. Tamajón \& R. Pinilla, COFC 22769.

Este taxon, cuya distribución conocida hasta el momento se restringía a Sierra Norte y Litoral (Devesa, 1987), se cita aquí por primera vez para la Comarca de Los Pedroches. Se ha recolectado creciendo en el sotobosque de fresnedas. Rara.

\section{Aphanes cornucopiodes Lag.}

CÓRDOBA: Hornachuelos, Finca «Aljabaras de Cárdenas», UH-10, 6-5-93, R. Tamajón \& R. Pinilla, COFC 22770.

Nueva población de esta especie de distribución restringida en Andalucía Occidental. En Sierra Norte cordobesa la especie se había localizado previamente en la zona centromeridional, estando también presente en Aracena, Sierra Norte sevillana, Subbética y Grazalema (Devesa, 1987). Escasa y localizada.

Anthyllis citysoides L.

CÓRDOBA: Hornachuelos, Finca «La Alcaidía», UG-09, 26-12-91, R. Tamajón \& R. Pinilla, COFC 21313.

Se trata de la primera cita de esta especie para la comarca de Sierra Norte, estando citada con anterioridad de las comarcas: Litoral, Campiñas Baja y Alta, Subbética, Grazalema y Algeciras (Domínguez, 1987). Se ha localizado en matorrales sobre sustratos calcáreos. Escasa y localizada.

\section{Hippocrepis ciliata Willd.}

CÓRDOBA: Almodóvar, Río Guadiato, UG18, 11-5-93, R. Tamajón, R. Pinilla \& A. Jiménez, COFC 22673.

Nueva cita para Sierra Norte. Hasta el momento se había citado en Condado-Aljarafe, Campiña de Huelva, Litoral, Marismas, Alcores, Vega, Campiñas (Alta y Baja), Subbética, Algeciras y Grazalema (Domínguez, 1987). En nuestro territorio es bastante rara, habiéndose recolectado creciendo sobre sustratos calcáreos.

\section{Trifolium dubium Sibth.}

CÓRDOBA: Hornachuelos, Finca «Mosqueros», UH-90, 22-4-93, R. Tamajón \& R. Pinilla, COFC 22683; Villaviciosa, "Cabeza Aguda», UH-10, 10-6-93, R. Tamajón \& R. Pinilla, COFC 21119.

Primera cita para Sierra Norte. Hasta el 
momento sólo había sido citado de Pedroches, Litoral, Marisma y Algeciras (Ruíz de Clavijo, 1987). No obstante, Cano \& González (1992) lo citan de Córdoba, sin especificar más, en su revisión sobre la flora de Sierra Morena. Se ha localizado creciendo en vallicares sobre sustratos silíceos. Poco frecuente.

\section{Medicago littoralis Rohde ex Loisel}

CÓRDOBA: Almodóvar, Río Guadiato, aguas abajo de la presa del Embalse de la Breña, UG-18, 11-5-93, R. Tamajón, R. Pinilla \& A. Jiménez,

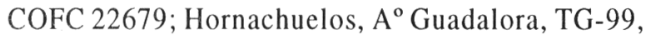
13-5-93, R. Tamajón \& R. Pinilla, COFC 22681.

Nueva cita para Sierra Norte. Con anterioridad se conocía de Alcores, Litoral, Marisma, Subbética gaditana, Grazalema, Algeciras y Vega (Romero Zarco, 1987). En el ámbito de Sierra Morena, fuera de Andalucía Occidental, sólo se conoce de la provincia de Badajoz (Cano \& González, 1992). Poco frecuente.

\section{Trigonella ovalis Boiss.}

CÓRDOBA: Hornachuelos, alrededores, UG08, 1-6-94, R. Tamajón \& R. Pinilla, COFC 24407.

Este taxon se cita aquí por primera vez para la comarca Sierra Norte. Con anterioridad el área conocida para esta especie se restringía a las comarcas Subbética cordobesa y Grazalema (Domínguez, 1987). La única población localizada, muy pequeña, se sitúa en los claros de un acebuchallentiscar con palmito, sobre sustratos calcáreos del Cámbrico Inferior, en el piso termomediterráneo superior. Muy rara.

\section{Ononis mollis Savi.}

CÓRDOBA: Almodóvar, Río Guadiato, aguas abajo de la presa del Embalse de la Breña, UG-18, 11-5-93, R. Tamajón, R. Pinilla \& A. Jiménez, COFC 22674; «Cuevas Altas», UG-29, 11-5-94, R. Tamajón, R. Pinilla \& J. M. Muñoz, COFC 24413; "Vereda de la Porrada», UG-20, 12-5-94, R. Tamajón, R. Pinilla \& J. M. Muñoz, COFC 24412; Córdoba, «Cerro del Trigo», UH-20, 3-6-93, R. Tamajón \& R. Pinilla, COFC 21120; Villafranca, Carretera Villafranca-Adamuz, UH-60, 11-5-94, R. Tamajón, R. Pinilla \& J. Muñoz, COFC 24414; Posadas, Finca «La Torre», UG-19, 26-5-94, R. Tamajón \& R. Pinilla, COFC 24415.

Primera cita de esta especie para la comarca de Sierra Norte. Previamente estaba citada del Litoral gaditano, Subbética, Alcores y Grazalema (Devesa, 1987). Se ha recolectado en tomillares sobre sustratos calcáreos miocénicos. Localizada y puntualmente abundante.

\section{Ulex eriocladus $\mathrm{C}$. Vicioso}

CÓRDOBA: Hornachuelos, Finca «San Calixto», TH-90, 16-12-91, R. Tamajón \& R. Pinilla, COFC 21579; Finca «Canónigos», TG-99, 26-2-92, R. Tamajón \& M. Carrasco, COFC 21580; Finca «Rayos Altos», UG-09, 27-2-92, R. Tamajón \& M. Carrasco, COFC 21581.

Citado hasta el momento en Aracena, Andévalo, Campiña de Huelva, Condado-Aljarafe y Litoral de Huelva (Rivas Martínez \& Cubas, 1987), ésta es la primera cita de este endemismo aracenopacense para Sierra Norte cordobesa. Esta población es la más oriental de todo su área de distribución ibérica, y por tanto, se trata de un área finícola. En el territorio se localiza sobre sustratos silíceos, en el piso mesomediterráneo inferior, formando parte de jarales pringosos o bien en dehesas invadidas por matorral serial. Localmente abundante.

\section{Astragalus epiglottis L. subsp. epiglottis}

CÓRDOBA, Hornachuelos, alrededores del pueblo, UG-08, 24-5-93, R. Tamajón \& R. Pinilla, COFC 22678, Carretera Hornachuelos-San Calixto, UG-09, 26-4-94, R. Tamajón \& R. Pinilla, COFC 22676.

Primera cita al norte del Guadalquivir, en concreto para Sierra Norte, estando citada previamente en la Subbética y en las dos Campiñas, Alta y Baja (Domínguez, 1987). Se ha localizado en pastizales sobre sustratos margosos y calcáreos miocénicos. Rara.

\section{Astragalus glaux L.}

CÓRDOBA, Hornachuelos, alrededores, UG08, 4-5-93, R. Tamajón \& R. Pinilla, COFC 22677.

Nueva población dentro de la comarca de Sierra Norte cordobesa. Su interés reside en que hasta el momento sólo se conocía de ciertas localidades de la Subbética Cordobesa (Muñoz \& Domínguez, 1985, Domínguez, 1987), así como de una localidad de Sierra Norte, «Sierra Palacios», Bélmez, UH-03 (Varela, Domínguez \& Ruíz de Clavijo, 1981), en cualquier caso bastante distante de la que aquí se cita. Se trata de un taxon muy raro en el territorio, 
habiéndose herborizado en pastizales sobre sustratos calcáreos.

Lythrum borysthenicum (Schrank) Litv. in Majevski CÓRDOBA: Villaviciosa, $\mathrm{A}^{\circ}$ Pajaroncillo, UH-10, 10-6-93, R. Tamajón \& R. Pinilla, COFC 22775.

Primera cita de esta especie para Sierra Norte. Las poblaciones anteriormente conocidas se localizan en Andévalo, Campiña de Huelva, Condado-Aljarafe, Litoral onubense y Marisma (Silvestre, 1987). Posteriormente ha sido recolectada en zonas húmedas de las comarcas del Zújar y Los Pedroches, donde es bastante frecuente (Jiménez \& Ruíz de Clavijo, 1990). Se presenta muy localizada y en pequeño número en comunidades sobre suelos silíceos.

\section{Lythrum portula (L.) D.A. Webb}

CÓRDOBA: Villaviciosa, $\mathrm{A}^{\circ}$ Pajaroncillo, UH-10, 10-6-93, R. Tamajón \& R. Pinilla, COFC 22777.

Con esta cita el área de distribución de esta especie, conocida hasta ahora de Aracena y parte de Algeciras (Silvestre, 1987), se amplía a la comarca de Sierra Norte. Ha sido recolectada en suelos húmedos o encharcados, sobre sustratos silíceos. Escasa y localizada.

\section{Osyris quadripartita Salzm. ex Decne}

CÓRDOBA: Hornachuelos, «Dehesa de Las Piedras", Finca "Cuchillares", TG-98, 18-12-91, J. M. Muñoz \& R. Tamajón, COFC 21194; Finca «Mosqueros», TH-90, 26-12-91, R. Tamajón \& R. Pinilla, COFC 21176; Finca «El Aguila», TG-99, 31-1-92, R. Tamajón, R. Pinilla \& M. Carrasco, COFC 21188; Finca «Aljabaras de Cárdenas», UH10, 25-2-92, R. Tamajón \& M. Carrasco, COFC 21179; «El Seminario de Los Angeles», UG-09, 17 12-92, R. Pinilla \& J.M. Muñoz, COFC 21195; Posadas, "Colada de Los Calamones», UG-19, 8-794, R. Tamajón, R. Pinilla, J. M. Muñoz \& M. Moral, COFC 24416; Pozoblanco, «Sierra del Castaño», UH-42, 23-3-95, R. Pinilla \& J.M. Delgado, COFC 24419; Obejo, «Cerro del Conjuro», UH-42, 6-4-95, R. Pinilla \& J. M. Delgado, COFC 24417.

Aunque no se trata de la primera cita de esta especie para la Sierra Norte cordobesa (Jiménez \& Ruíz de Clavijo, 1990) se amplía notablemente su areal. Hasta el momento sólo se conocía, aparte de la comarca mencionada, de Algeciras, Grazalema y Litoral (Ruíz de Clavijo, 1987). Se trata de una especie indiferente edáfica, prefiriendo litosuelos y laderas más o menos rocosas, en los pisos termomediterráneo superior y mesomediterráneo inferior. Se ha localizado en un amplio rango de comunidades vegetales, pero sobre todo en jarales termófilos, jarales-brézales y acebuchales. Localmente abundante.

Erodium aethiopicum (Lam.) Brumh. \& Thell. in Thell. subsp. aethiopicum

CÓRDOBA: Villaviciosa, Río Guadiatillo, «Cerro del Trigo», UH-20, 3-6-93, R. Tamajón \& R. Pinilla, COFC 22810; Almodóvar, proximidades del Embalse de la Breña, UG-29, R. Tamajón \& R. Pinilla, COFC 22808.

Se trata de la primera cita de esta subespecie para Sierra Norte. Hasta el momento sólo era conocida del Litoral y Campiña Baja (Devesa, 1987). En el territorio se ha localizado en pastos sobre suelos arenosos, en bordes de cursos fluviales. Rara.

\section{Bupleurum rigidum L. subsp. rigidum}

CÓRDOBA: Almodóvar, Río Guadiato, "Umbría de Valdeinfierno", UG-29, 11-6-94, R. Pinilla \& M. Moral, COFC 24410.

Nueva cita para la comarca de Sierra Norte, de gran interés porque el área de distribución conocida de este taxon se restringía exclusivamente a la Subbética (García Martín, 1987). Se ha recolectado en acebuchales-lentiscares, sobre sustratos volcánicos (queratófidos y espilitas). Muy rara.

\section{Ferula communis L.}

CÓRDOBA: Hornachuelos, Finca «Rayos Altos», UH-00, 13-4-93, R. Tamajón \& R. Pinilla, COFC 24396; Villaviciosa, $A^{\circ}$ Pajaroncillo, UH10, 10-6-93, R. Tamajón \& R. Pinilla, COFC 24397; Río Retortillo, 24-6-93, R. Tamajón \& R. Pinilla, COFC 24398.

Primera cita para la comarca de Sierra Norte de Córdoba. Previamente estaba citada de Aracena, Sierra Norte sevillana, Campiña Alta, Subbética y Grazalema (García Martín, 1987). Posteriormente fue citada del NO de Los Pedroches -Sierra de Santa Eufemia- (Jiménez \& Ruíz de Clavijo, 1990). Se desarrolla en comunidades muy diversas tales como alisedas, acebuchales y agracejales, entre otras, 
tanto sobre sustratos calcáreos como silíceos, en los pisos termomediterráneo superior y mesomediterráneo inferior. Localmente abundante.

\section{Convolvulus siculus L. subsp. siculus}

CÓRDOBA: Córdoba, cercanías del Arroyo Pedroches, UH-40, 21-4-94, J. M. Muñoz, COFC 24395.

Esta cita de Sierra Norte cordobesa amplía el área de distribución de este taxon en la comarca de Sierra Norte, puesto que estaba ya citado puntualmente- de la provincia de Sevilla. Otras comarcas donde se presenta son: Aracena, Campiña Alta, Subbética, Grazalema y Algeciras (Valdés, 1987) y Zújar (Jiménez \& Ruíz de Clavijo, 1990). Al igual que en el resto de su área de distribución con excepción de la comarca del Zújar- presenta un comportamiento edáfico calcícola. Se trata de una especie rara en el territorio, donde se ha herborizado en cerrillares sobre litosuelos de la serie carbonatada del Cámbrico Inferior, con fuertes pendientes. Rara.

\section{Lamium purpureum $\mathrm{L}$}

CÓRDOBA: Torrecampo, Río Guadalmez, UH-56, 18-3-94, R. Tamajón \& R. Pinilla, COFC 22792.

Esta rara especie tiene una distribución muy restringida en Andalucía Occidental: Aracena, Sierra Norte de Sevilla y Campiña Alta de Córdoba (Ubera 1987). Esta localidad constituye por tanto la primera cita para Los Pedroches. Se ha encontrado en escaso número en el estrato herbáceo de alisedas.

\section{Teucrium scorodonia L. subsp. scorodonia}

CÓRDOBA: Villaviciosa, Arroyo del Castaño, UH-10, 13-6-94, R. Tamajón \& R. Pinilla, COFC 24408.

Nueva cita para la comarca de Sierra Norte cordobesa. Con anterioridad se conocía de Aracena y Sierra Norte sevillana (Devesa, 1987), así como de Los Pedroches (Jiménez \& Ruíz de Clavijo, 1990). Se ha herborizado en el sotobosque de encinares mesomediterráneos. Rara.

Linaria oblongifolia (Boiss.) Boiss. \& Reuter subsp. haenseleri (Boiss. \& Reuter) Valdés

CÓRDOBA: Hornachuelos, alrededores del pueblo, Finca «Cuchillares», UG-08, 4-3-93, R. Pinilla \& R. Tamajón, COFC 21367.

Se trata de una población muy reducida y localizada en la porción occidental de Sierra Norte cordobesa, en contacto con la Vega. Hasta el momento, las únicas poblaciones conocidas de este este endemismo bético en Sierra Norte cordobesa se localizaban en la mitad oriental (Valdés, 1987). En el territorio se halla muy localizada, habiéndose herborizado sobre sustratos calcáreos miocénicos, en el piso termomediterráneo superior, formando parte de pastizales terofíticos.

\section{Kickxia cirrhosa (L.) Fritsch}

CÓRDOBA: Hornachuelos, Finca «Rayos Bajos», TG-90, 11-6-93, R. Tamajón \& R. Pinilla, COFC 22800.

Nueva cita para Sierra Norte. Se trata de un taxon interesante, puesto que posee una distribución muy restringida en Andalucía Occidental, a saber: Andévalo, Litoral, Campiña Baja gaditana y Algeciras (Valdés, 1987). Se trata de una especie muy rara en el territorio, que se ha localizado en vallicares sobre sustratos silíceos, en suelos húmedos.

\section{Globularia alypum L.}

CÓRDOBA: Hornachuelos, Finca «Cabalgaderos de San Bernardo», TG-99, 19-1291, R. Tamajón \& R. Pinilla, COFC 21426; Finca «La Alcaidía», UG-09, 26-12-91, R. Tamajón \& R. Pinilla, COFC 21425.

Con esta primera cita para Sierra Norte se amplía hacia el norte el área de distribución de este taxón, restringido hasta el momento al Litoral gaditano, Campiña Alta gaditana y sevillana, y parte de Grazalema y Algeciras (Valdés, 1987), habiéndose citado posteriormente para la Campiña Alta cordobesa (García Montoya \& Muñoz, 1990). No se trata de la primera cita para la Cordillera Mariánica en su conjunto, ya que en Sierra Morena Oriental (comarca de Despeñaperros) se conoce desde hace tiempo una población (Rivas Goday \& Bellot, 1946). Se ha localizado sobre sustratos calcáreos miocénicos y cámbricos del piso termomediterráneo superior, en diversos matorrales (jarales y acebuchales). Escasa y localizada.

Lonicera periclymenum L. subsp. hispanica (Boiss. \& Reuter) Nyman CÓRDOBA: Santa María de Trassierra, A ${ }^{\circ}$ del Molino, UH-30, 9-4-95, R. Pinilla, COFC 24406.

Esta especie no estaba citada para la comarca 
Sierra Norte. Era conocida previamente de Aracena, Litoral, Grazalema y Algeciras (García Martín, 1987), y posteriormente de Los Pedroches (Jiménez \& Ruíz de Clavijo, 1990). Poco frecuente.

\section{Matricaria aurea (Loefl.) Schultz Bip}

CÓRDOBA: Fuente Obejuna, «Cerro del Castillo», TH-92, 10-3-95, R. Tamajón \& R. Pulido, COFC 24405.

Nueva cita para Sierra Norte. Esta especie, considerada como muy rara, se había citado previamente de Los Pędroches y de La Vega (Talavera, 1987). La población localizada se presenta en majadales muy pisoteados, en compañía de Gymnostyles stolonifera (Brot.) Tutin.

Glossopappus macrotus (Durieu) Briq., in Burnat CÓRDOBA: Hornachuelos, alrededores del pueblo, Finca «Cuchillares», UG-08, 4-5-93, COFC 21496.

Primera cita para Sierra Norte, era conocida de la Vega, Alcores, Campiña (Alta y Baja), Subbética y Grazalema (Talavera, 1987). Se presenta en herbazales subnitrófilos, sobre sustratos calcáreos. Poco frecuente.

\section{Gymnostyles stolonifera (Brot.) Tutin}

CÓRDOBA: Fuente Obejuna, "Cerro del Castillo», TH-92, 10-3-95, R. Tamajón \& R. Pulido, COFC 24399.

Nueva cita de este neófito para la Sierra Norte cordobesa. Hasta el momento era conocido de Aracena, Andévalo, Campiña de Huelva, CondadoAljarafe, Vega sevillana, Litoral, Marisma, Grazalema y Algeciras (Talavera, 1987). Localmente abundante, se comporta como indiferente edáfica. Se ha herborizado en pastos pisoteados.

Aetheorhiza bulbosa (L.) Cass. CÓRDOBA: Posadas, Arroyo Guadalvacarejo, UG-19, 6-5-93, R. Tamajón \& R. Pinilla, COFC 22747. Nueva cita para la comarca de Sierra Norte. Hasta el momento sólo se conocía de Aracena, Alcores, Litoral, Campiña Alta, Subbética, Grazalema y Algeciras (Talavera, 1987). Este taxon, muy raro en el territorio, se ha localizado sobre sustratos silíceos, en el sotobosque de fresnedas.

Leuzea conifera (L.) DC. in Lam. \& DC. CÓRDOBA: Hornachuelos, Finca «Olivar de
Santa María», TG-99, 8-7-94, R. Tamajón, R. Pinilla \& J. M. Muñoz, COFC 24402.

Nueva cita para Sierra Norte. Previamente estaba citada de Aracena, Litoral, Campiña Alta, Subbética, Grazalema y Algeciras (Gallego, 1987). Se presenta sobre sustratos calcáreos cámbricos. Muy rara.

\section{Staehelina dubia L.}

CÓRDOBA: Hornachuelos, Finca «Los Arenales», UG- 99 , 17-12-91, J. M. Muñoz \& R. Pinilla, COFC 21527.

Primera cita para Sierra Norte. Hasła el momento sólo se conocía de las siguientes comarcas: Campiña Alta, Subbética, Grazalema y Litoral (Talavera, 1987). Se ha encontrado, en pequeño número, creciendo en matorrales sobre sustratos calcáreos. Muy rara.

Juncus tenageia Ehrh. ex L. fil.

CÓRDOBA: Villaviciosa, A ${ }^{\circ}$ Pajaroncillo, UH10, 10-6-93, R. Tamajón \& R. Pinilla, COFC 22783.

Se trata de la primera cita para Sierra Norte cordobesa. Hasta el momento estaba citado de Sierra Norte sevillana, Andévalo, Campiña de Huelva, Litoral, Algeciras (Fernández Carvajal, 1987) y Los Pedroches (Jiménez \& Ruíz de Clavijo, 1990). Se desarrolla sobre sustratos silíceos, en suelos encharcados. Rara.

\section{Scirpus lacustris $\mathrm{L}$.}

CÓRDOBA: Santa María de Trassierra, Río Guadiato, UH-20, 9-4-95; R. Pinilla, COFC 24418.

Nueva cita para Sierra Norte de Córdoba. Con antelación se conocía sólo de Sierra Norte sevillana, Condado-Aljarafe y Litoral onubense (Gallego, 1987); más recientemente se ha citado de las comarcas del Zújar y de Los Pedroches (Jiménez \& Ruíz de Clavijo, 1990). Especie muy localizada, en medios inundados.

\section{Hainardia cylindrica (Willd.) Greuter}

CÓRDOBA: Villaviciosa, Finca «El Escoboso», UH-10, 5-6-92, R. Tamajón \& R. Pinilla, COFC 21301.

Nueva cita para Sierra Norte. Hasta ahora se conocía en las comarcas de: Vega, Litoral, Marisma, Campiña (Baja y Alta), Subbética, Grazalema y Algeciras (Talavera, 1987). En pastos sobre sustratos silíceos, muy escasa y localizada. 


\section{Holcus mollis L. subsp. mollis}

CÓRDOBA: Hornachuelos, Finca «La Loma», TH-80, 18-6-92, R. Tamajón \& R. Pinilla, COFC 21246 .

Esta es la primera cita para la comarca de Sierra Norte. Hasta el momento sólo era conocido este taxon de Aracena y Algeciras (Romero Zarco, 1987). Se ha localizado en praderas-juncales, sobre sustratos pizarrosos del Cámbrico Inferior. Rara.

\section{Agrostis tenerrima Trin.}

CÓRDOBA: Hornachuelos, Loma del Bayón, UH-00, 2-6-92, R. Tamajón \& M. Carrasco, COFC 21269; Villaviciosa de Córdoba, Loma de los Jarales, UH-10, 30-2-92, R. Tamajón \& R. Pinilla, COFC 21268.

Primera cita de este taxon para la comarca de Sierra Norte. Previamente estaba citado de Aracena, Andévalo, Campiña de Huelva, Litoral, Grazalema y Algeciras (Romero Zarco, 1987), así como de la comarca del Zújar, (Jiménez \& Ruíz de Clavijo, 1990). Se ha herborizado en pastizales terofíticos, en claros de jaral-brezal, sobre sustratos silíceos (pizarras y esquistos cámbricos). Escaso y localizado.

\section{Brachypodium retusum (Pers.) Beauv.}

CÓRDOBA, Hornachuelos, Finca «El Asiento», TG-99, R. Tamajón \& R. Pinilla, COFC 24388.

Se trata de una nueva población en Sierra Norte cordobesa, con lo que el área de distribución conocida en el seno de dicha comarca se amplía notablemente, ya que hasta el momento sólo se había citado de una pequeña porción de la mitad oriental (Talavera, 1987). Se ha herborizado en matorrales sobre sustratos calcáreos cámbricos. Escasa y localizada.

\section{Hordeum geniculatum All.}

CÓRDOBA: Almodóvar, Finca «Cabeza Pedro Bajo», UG-29, 27-4-93, R. Tamajón \& R. Pinilla, COFC 21111; Córdoba, Río Guadalquivir, «Sotos de la Albolafia», UG-49, 30-4-95, R. Tamajón \& R. Pinilla, COFC 24403.

Nueva cita para Sierra Norte y Vega. La distribución conocida hasta el momento se reducía a Pedroches, Litoral, Marisma, Subbética cordobesa, Grazalema y Algeciras (Talavera, 1987). Se trata de una especie rara en el territorio, donde se desarrolla en herbazales sobre suelos más o menos húmedos.

\section{Stipa gigantea Link.}

CÓRDOBA: Córdoba, «Cerro de Pedro López», UH-30, R. Pinilla, COFC 24386.

Nueva cita para la comarca de Sierra Norte. Hasta el momento se conocía de Aracena, Litoral, Alcores, Campiña (Alta y Baja), Subbética, Grazalema y Algeciras (Talavera, 1987), así como de Los Pedroches (Jiménez \& Ruíz de Clavijo, 1990). La población aquí citada, se desarrolla sobre sustratos graníticos. Localmente abundante.

\section{Aristida coerulescens Desf.}

CÓRDOBA: Córdoba, Cercanías del A Pedroches, UH-40, 21-4-94, J. M. Muñoz, COFC 24389.

La población localizada en Sierra Norte cordobesa constituye la primera cita de este taxon para Andalucía Occidental. Esta especie es de óptimo Murciano-Almeriense, bajo ombroclima semiárido, si bien puede alcanzar áreas alpujarreño-gadorenses y setabenses (Alcaraz, 1984). La presencia de esta especie de forma finícola en nuestro territorio es destacable, puesto que, a pesar de existir algunos factores ecológicos favorables -piso termomediterráneo, sustratos calcáreos- el ombroclima es subhúmedo inferior, y su área de distribución óptima está bastante alejada de esta población. Es una especie muy rara, habiéndose herborizado formando parte de cerrillares sobre sustratos calcáreos del Cámbrico Inferior.

\section{Piptatherum thomasii (Duby) Kunth}

CÓRDOBA: Hornachuelos, Finca «Rincón Alto», TG-99, 5-2-92, R. Pinilla \& J.M. Quero, COFC 21295; Río Guazulema, 29-6-93, R. Tamajón \& R. Pinilla, COFC 21272; A ${ }^{\circ}$ de Mahoma, TG-98, 3-6-94, R. Tamajón \& R. Pinilla, COFC 24393; Posadas, Carretera Posadas-Villaviciosa, UG-19, 13-6-94, R. Tamajón \& R. Pinilla, COFC 24392; A ${ }^{\circ}$ Guadalbaida, UG-18, 16-3-94, R. Tamajón \& R. Pinilla, COFC 24385

Esta es la primera cita para Sierra Norte cordobesa, habiéndose citado con anterioridad de Sierra Norte sevillana, Subbética, Grazalema y Algeciras (Devesa, 1987). Localmente abundadente, se ha localizado en el territorio sobre sustratos silíceos y calcáreos, en herbazales vivaces y en el estrato herbáceo de fresnedas y tamujares, entre otras comunidades. 
Piptatherum paradoxum (L.) Beauv.

CÓRDOBA: Hornachuelos, «Monte Alto», TG-98, 3-6-94, R. Tamajón \& R. Pinilla, COFC 24391; Córdoba, Sierra de Córdoba, en la cercanía del «Cortijo El Melgarejo», UH-30, 14-2-95, R. Tamajón \& R. Pulido, COFC 24390.

Notable cita para Sierra Norte, puesto que hasta el momento sólo se conocía de comarcas bastante alejadas, Subbética y Grazalema (Devesa, 1987). Esta especie, localmente abundante, se ha recolectado en el estrato herbáceo de encinares y alcornocales, sobre sustratos calcáreos del Cámbrico y del Mioceno.

Asphodelus albus Miller, subsp. villarsii (Verlot ex Billot) B. K. Richardson \& Smythies

CÓRDOBA: Hornachuelos, "Mesas del Bembézar», UH-00, 12-2-92, R. Pinilla, COFC 21151; «Finca Cuchillares», UG-09, 4-3-93, R. Tamajón \& R. Pinilla, COFC 22766; Finca «Cabalgaderos de San Bernardo», TG-99, 25-5-94, R. Tamajón \& R. Pinilla, COFC 22763.

Estas citas son las primeras para la comarca de Sierra Norte, conociéndose con anterioridad tan sólo de Aracena, Subbética, Grazalema y Algeciras (Valdés, 1987). Ha sido citada también en Los Pedroches, donde es poco frecuente (Jiménez \& Ruíz de Clavijo, 1990). En nuestro territorio es relativamente escasa, localizándose sobre sustratos calcáreos del Cámbrico Inferior.

\section{Iris pseudacorus $\mathrm{L}$.}

CÓRDOBA: Córdoba, Río Guadiato, «Umbría de Perchas», UG-29, R. Tamajón \& R. Pinilla, COFC 22767.

Nueva cita para la comarca de Sierra Norte, ya que sólo se conocía de: Zújar, Andévalo, Litoral, Marisma, Grazalema y Algeciras (Valdés, 1987). Se ha herborizado formando partes de comunidades higrófilas, así como en saucedas. Localmente abundante.

\section{BIBLIOGRAFÍA}

ALCARAZ, F. J. -1984- Flora y Vegetación del NE de Murcia. Publicaciones de la Universidad de Murcia. Murcia.

CABEZUDO, B. y A.E. SALVO-1987- Cheilanthes, in B. VALDÉS et al. (eds.) Flora Vascular de Andalucía Occidental 1: 49-51. Editorial Ketres. Barcelona.
CANO, E. y A. GONZÁLEZ -1992-Estudios básicos para el conocimiento de la flora de Sierra Morena. Facultad de Ciencias Experimentales de Jaén. Jaén.

CLEMENTE, M. y B. HIDALGO -1987- Lepidium in B. VALDÉS et al. (eds.) Flora Vascular de Andalucia Occidental 1: 420-423. Editorial Ketres. Barcelona.

DEVESA, J. A. - 1987- Illecebrum, Loeflingia, Rosa, Sanguisorba, Aphanes, Ononis, Erodium, Teucrium, Piptatherum, in B. VALDÉS et al. (eds.) Flora Vascular de Andalucía Occidental $1: 213,215-216,2: 26-29,30-33,37-38,132-$ 154, 275-284, 458-469, 3: 385-387. Editorial Ketres. Barcelona.

DOMÍNGUEZ, E. -1987- Anthyllis, Hippocrepis, Trigonella, Astragalus, in B. VALDÉS et al. (eds.) Flora Vascular de Andalucía Occidental 2: 81-86, 93-95, 127-129, 182-189. Editorial Ketres. Barcelona.

FERNÁNDEZ CARVAJAL, M. C.-1987- Juncus, in B. VALDÉS et al. (eds.) Flora Vascular de Andalucía Occidental 3: 213-228. Editorial Ketres. Barcelona.

FERNÁNDEZ, P. y E. DOMÍNGUEZ -1991Análisis corológico del Parque Natural de la Sierra de Hornachuelos. Actas de la $1^{\text {a }}$ Reunión sobre el Medio Ambiente en Andalucía. Facultad de Ciencias-Jardín Botánico. Córdoba.

GALLEGO, M. J. - 1987- Tuberaria, Leuzea, Scirpus, in B. VALDÉS et al. (eds.) Flora Vascular de Andalucia Occidental 1: 344-346, 3: 161, 229 . 235. Editorial Ketres. Barcelona.

GARCÍA MARTÍN, F. - 1987- Bupleurum, Ferula y Lonicera, in B. VALDÉS et al. (eds.) Flora Vascular de Andalucía Occidental 2: 308-314, 321, 597-598. Editorial Ketres. Barcelona.

GARCÍA MONTOYA, F. y J. M. MUÑOZ -1990Novedades corológicas para la Flora de Andalucía Occidental. Lagascalia 16 (1): 146-168.

JIMÉNEZ, M. y E. RUÍZ DE CLAVIJO -1990Novedades corológicas para la Flora de Andalucía Occidental. Lagascalia 16(1): 132-145.

MUÑOZ, J. M. y E. DOMÍNGUEZ - 1985-Catálogo florístico del Sur de la Provincia de Córdoba. Córdoba.

RIVAS GODAY, S. y F. BELLOT -1946- Estudios sobre la vegetación y flora de la comarca Despeñaperros-Santa Elena. Anales Jardín Botánico de Madrid 6: 93-215. 
RIVAS MARTÍNEZ, S. y P. CUBAS -1987- Ulex, in B. VALDÉS et al. (eds.) Flora Vascular de Andalucía Occidental 2: 165-169. Editorial Ketres. Barcelona.

ROMERO ZARCO, C. -1987- Medicago, Holcus, Agrostis, in Flora Vascular de Andalucia Occidental 2: 117-127, 3: 331-333, 336-341. Editorial Ketres. Barcelona.

RUÍZ DE CLAVIJO, E. - 1987- Trifolium, Osyris, in B. VALDÉS et al. (eds.) Flora Vascular de Andalucía Occidental 2: 97-116, 211-212. Editorial Ketres. Barcelona.

SILVESTRE, S. - 1987- Lythrum, in B. VALDÉS et al. (eds.) Flora Vascular de Andalucía Occidental 2: 193-198. Editorial Ketres. Barcelona.

TALAVERA, S. -1987- Moehringia, Matricaria, Glossopappus, Gymnostyles, Aetheorhiza, Staehelina, Hainardia, Brachypodium, Hordeum, Stipa, in B. VALDÉS et al. (eds.) Flora Vascular de Andalucia Occidental 1 : 231, 3: 50-51, 62-63, 69, 91-92, 175, 299, 369-372, 378-380, 381-384. Editorial Ketres. Barcelona. UBERA, J. L. -1987- Lamium, in B. VALDÉS et al. Flora Vascular de Andalucía Occidental 2: 409411. Editorial Ketres. Barcelona.
VALDÉS, B. -1987- Convolvulus, Linaria, Kickxia, Globularia, Asphodelus, Iris, in B. VALDÉS et al. (eds.) Flora Vascular de Andalucía Occidental 2: 363-369, 508-522, 525-528, 548-549, 3: 425-427, 477-482. Editorial Ketres. Barcelona.

VALDÉS, B., S. TALAVERA y E. FERNÁNDEZ GALIANO (eds.) -1987- Flora Vascular de Andalucía Occidental. 3 vols. Editorial Ketres. Barcelona.

VARELA, J. A., E. DOMÍNGUEZ y E. RUÍZ DE CLAVIJO -1981- Notas sobre la Flora de Córdoba. I. Algunas especies interesantes de los tramos medio e inferior del valle del Río Guadiato. Anales Jardín Botánico de Madrid, 38(1): 320-322.

Aceptado para su publicación en Abril de 1998

Dirección de los autores. Departamento de Biología Vegetal y Ecología. Facultad de Ciencias. Universidad de Córdoba. 14004 - Córdoba.

\section{CISTUS LADANIFER L. SUBSP. AFRICANUS DANS., UN NUEVO TAXON PARA ANDALUCÍA OCCIDENTAL}

Pablo GARCÍA MURILLO y Manuela PALACIOS

Cistus ladanifer subsp. africanus Dans., a new taxon in West Andalusia

Palabras clave. Cistaceae, Cistus, Andalucía Occidental.

Key Words. Cistaceae, Cistus, West Andalusia.

En el tratamiento de Cistus L. que realizaron Demoly y Montserrat para la Flora Ibérica (1993), reconocieron tres subespecies dentro de C. ladanifer L.: la subsp. ladanifer, abundante en el oeste de la Península, la subsp. africanus Dans., indicada sólo para Málaga y la subsp. sulcatus (Demoly) P. Monts., referida al Algarve portugués. 